\title{
Contribution to the theory of limiting factors
}

\section{F. GÓRSKI}

(Wpłynęło dn. 20. XII. 1951 r.)

The theory of limiting factors, formulated in 1905 by F. F. B l a c k m a n n, is an attempt to determine more precisely the interdependence of factors controlling physiological processes, especially the carbon dioxyde assimilation. The theory states that ,when a process is conditioned as to its rapidity by a number of separate factors, the rate of the process is limited by the pace of the "slowest " factor" (B l a c k m a n n, 1905). If moreover we assume that the rate of the process is proportional to the intensity of the slowest factor, then it follows from the theory that on a graph the curve relating the rate to the intensity of the factor in question takes the form of two intersecting segments. Thus in the case of light the first segment represents the rate of photosynthesis for such intensities of light for which light is the limiting factor, while the second, parallel to the $\mathrm{X}$ axis, corresponds to the photosynthetic activity for light intensities sufficiently high for some other factor to become the limiting agent. The value at which there is a transition from the limitation by one factor (the light) to the limitation by another factor is the transition value ( $\mathrm{v}$ a $\mathrm{n}$ d e n $\mathrm{H}$ o n e r t, 1930).

Blackmann's theory made the object of several investigations. For a review of their results the reader is referred to the monographs by $\mathrm{Stiles}$ (1926), S p o e h r (1926) and B r ill i a n t (1949) and the papers by $\mathrm{H}$ a r d e r (1920), T s c h e s n o k o v and B a zyrina (1930), van der Pa a u (1930), va n d e n H o n e r t (1932) and S m i th $(1937,1939)$. It can be stated however that at first sight there seems to be little agreement between the theory and the experimental results. In most cases instead of two intersecting segments, two short segments connected 
by a long arch are obtained. This kind of curve indicates that for a certain range of intensities not one but two factors limit the rate of assimilation - a conclusion which is inconsistent with the basic assumptions of the theory.

The cause of these deviations from the theory was sought in the inequality of the experimental conditions. It is tacitly assumed in the theory that at a given moment the value of the constant factors as well as of the changing factor must not only be constant but also equal in all assimilating cells. Let us suppose for instance that the $\mathrm{CO}_{2}$ concentration or the temperature vary in different parts of the plant. It is obvious then that light will be the limiting factor in one part of the plant, and in another the temperature or the dioxyde concentration and that for a certain range of light intensities not one but two factors will be limiting the assimilation rate. From these remarks we conclude that not only the constancy but also the uniformity of all the experimental factors, is an essential condition, for the application of Blackmann's principle.

In fact this condition is seldom fullfilled. Let us for instance consider an algal suspension in a manometric vessel illuminated from below. Owing to the absorption of the luminous energy by cell pigments the intensity of light diminishes gradually from the bottom to the surface of the suspension and has the same value only for the cells at the same level. In multicellular plants owing to diffusion obstacles not only the light intensity but also the carbon dioxyde concentration will vary from one part of the plant to another. Both va $\mathrm{n}$ d e $\mathrm{n}$ Hon e r $\mathrm{t}$ (1930) and $\mathrm{v}$ a $\mathrm{n}$ d e $\mathrm{r}$ P a a u w (1932) fully appreciated the importance of the uniformity of experimental conditions in their study on the validity of Blackmann's theory, and they made provision to eliminate the possible source of errors by reducing to one layer the thickness of the assimilating cells. Some of the experimental curves obtained by both of them are in good agreement with the shape required by the theory.

It is the purpose of this paper to corroborate the validity of Blackmann's theory in a different manner. Its aim is to demonstrate that the shapes and the equations of the experimental curves representing the rate of photosynthesis in relation to the light intensity or the carbon dioxyde concentration can be deduced from the principle of limiting factors, provided that the distribution of the light intensity in the assimilating plants or cells is taken into consideration. For this purpose the assimilation conditions existing in an algal suspension of the thickness $d$ illuminated from below (fig. 1) will be 

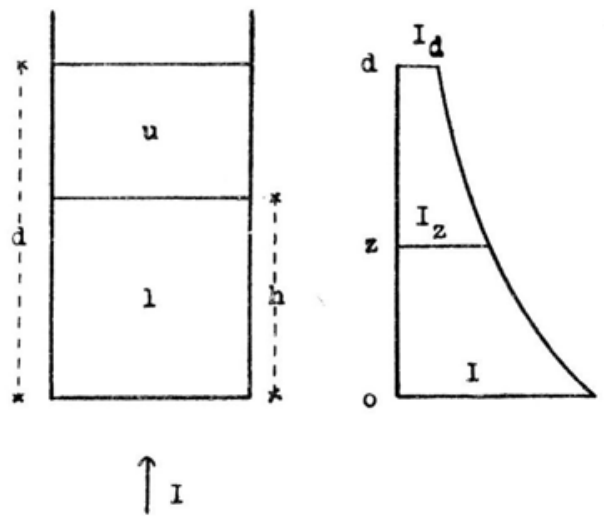

Fig. 1. (left) Algal suspension illuminated from below. I - light, d - thickness of the algal layer. $\mathrm{h}$ - height of the transition level. Light is the limiting factor in the upper (u) part, and the factor in excess in the lower (1) part of the suspension. (right) Decrease of the light intensity in the suspension. $\mathrm{I}$ - intensity of the incident light (at the bottom), $\mathrm{I}_{\mathrm{z}}$ and $\mathrm{I}_{d}$ - light intensities at the level $\mathrm{z}$ and at the surface of the suspension.

submitted to a closer examination. The light intensity $I_{z}$ at the level $z$ is given by the formula

$$
I_{z}=I e^{--k z},
$$

where $I$ is the intensity of the incident light and $k$ the light absorption constant. This relation can also be written:

$$
e^{k z}=\frac{l}{I_{z}}, \quad z=\frac{1}{k} \text { lognat } \frac{I}{I_{z}} .
$$

We will assume that the assimilation rate $d F$ of a very thin algal layer at the level $z$ obeys to Blackmann's principle i. e. increases proportionally to the light intensity $I_{z}$ as long as this intensity remains less than the transition value $I_{i}$ :

$$
d F=a I_{z} d z \quad \text { for } o \leqslant I_{z} \leqslant I_{t} .
$$

For light intensities higher than $I_{t}$ the assimilation rate $d F$ assumes a constant value independent of the light intensity:

$$
d F=a I_{t} d z \quad \text { for } I_{z} \geqslant I_{t} .
$$

In the algal suspension we may distinguish two layers: the first or lower extends from the bottom $(z=o)$ to lewel $z=h$ at which the 
light intensity has decreased to the transition value $I_{t}$. The height of the transition level is (from 3):

$$
h=\frac{1}{k} \operatorname{lgn} \frac{I}{I_{t}} .
$$

In the second or upper layer, extending from level $h$ to the surface $(z=d)$ of the suspension, the light intensity has at all levels values smaller than $I_{t}$. The rate of assimilation of the whole suspension is the sum of the rates of both the lower and the upper layers: $F=F_{1}+F_{2}$. In deducing them we may observe that the relations (5) and (4) are valid in the lower and upper layers respectively.

It follows from (5) that the rate $F_{1}$ is the sum of the rates of all the infinitesimal layers whose global thickness is $h$ :

$$
F_{l}=\int_{o}^{h} a I_{t} d z=\left.a I_{t} z\right|_{\bullet} ^{h}=a I_{t} h
$$

substituting for $h$ the value (6) we obtain:

$$
F_{1}=\frac{a I_{t}}{k} \operatorname{lgn} \frac{I}{I_{t}}
$$

Similarly $F_{2}$ is the sum of the rates of all the infinitesimal layers forming the upper part of the suspension. It follows from (4) and (1)

$$
F_{2}=\int_{h}^{d} a I_{z} d z=a \int_{h}^{d} I e^{-k z} d z=\left.\frac{-a I}{k} e^{-k z}\right|_{h} ^{d}=\frac{a I}{k}\left(e^{-k h}-e^{-k d}\right)
$$

and by (2):

$$
F_{2}=\frac{a I}{k}\left(\frac{I_{t}}{I}-\frac{1}{e^{k d}}\right)=\frac{a}{k}\left(I_{t}-\frac{I}{e^{k d}}\right)
$$

Thus the assimilation rate of the whole algal suspension expressed in terms of the light intensity is:

$$
\begin{gathered}
F=F_{1}+F_{2}=\frac{a I_{t}}{k} \operatorname{lgn} \frac{I}{I_{t}}+\frac{a}{k}\left(I_{t}-\frac{I}{e^{k d}}\right) \\
I_{t} \leqslant I \leqslant I_{t} e^{k \cdot d}
\end{gathered}
$$

Function (9) has a physiological meaning only for values of $I$ included between the limits $I_{t}$ and $I_{t} e^{k d}$. Indeed, it follows from (7) that 
when $I<I_{t}$ the value of $F_{1}$ is negative and $F_{1}$ is therefore deprived of physiological significance. Similarly it is apparent from equation (8) that $I$ must be smaller than $I_{t} e^{k d}$, because otherwise $F_{2}$ would assume a negative value. On the graph (fig. 2) representing function (9) only this part of the curve which lies between points $A$ and $B$ (the abscissae of which are $I_{t}$ and $I_{t} e^{k d}$ ) has a physiological significance. From (9) we may calculate the corresponding ordinates and thus obtain the following values for the coordinates of $A$ and $B$ :

$$
\begin{aligned}
& \text { point } A: I_{A}=I_{t}, \quad F_{A}=\frac{a}{k}\left(1-\frac{1}{e^{k d}}\right) I_{t}, \\
& \text { point } B: I_{B}=I_{t} e^{k d}, \quad F_{B}=a I_{t} d .
\end{aligned}
$$

Thus it becomes necessary to inquire as to the form cf $F$ corresponding to the values of $I$ below $I_{t}$ or above $I_{t} e^{k d}$. The condition $I<I_{t}$ signifies that the equation (4) $d F=a I_{z} d z$ is valid for every level of the suspension. Substituting in (4) for $l_{z}$ the value $I_{z}=I e^{-k z}$ (formula (1) and integrating between the limits o and $d$ we obtain:

$$
\begin{gathered}
F=\int_{o}^{d} a I e^{-k d} d z=\left.\frac{-a I}{k} e^{-k d}\right|_{o} ^{d}=\frac{-a I}{k}\left(e^{-k d}-1\right) \\
F=\frac{a}{k}\left(1-\frac{1}{e^{k d}}\right) I, \quad o \leqslant I \leqslant I_{t}
\end{gathered}
$$

Formula (11) represents the equation of a straight line passing through the origin and point $A$ on the curve. It follows from (11) that for small light intensities the assimilation rate is proportional to the light intensity.

When $I>I_{t} e^{k d}$ every layer of the suspension receives luminous energy in amounts superior to the transition value $I_{t}$. Indeed, it may be seen from (2) that for $I>I_{t} e^{k d}$ even at the surface we have $I_{d}>I_{t}$. We must therefore apply equation (5) to the whole suspension:

$$
F=\int_{0}^{d} a I_{t} d z=\left.a I_{t}\right|_{o} ^{d}=a I_{t} d, \quad I \geqslant I_{t} e^{k d} .
$$

For high light intensities the assimilation rate is independent of the light intensity. On the graph a line parallel to the $X$-axis passing through point $B\left(I_{t} e^{k d}, a I_{t} d\right)$ of the curve is obtained. 
The straight lines defined by the equations (11) and (12) do not intersect with curve (9) but are tangent to it at the points $A$ and $B$ respectively. The equation of the tangent to a curve $y=f(x)$ at the point $x, y$ is:

$$
Y-y=(X-x) f^{\prime}(x),
$$

where $Y$ and $X$ are the coordinates of the tangent. In our case

$$
y=f(I), \quad x=I, \quad f^{\prime}(x)=F^{\prime}=\frac{a I_{t}}{k I}-\frac{a}{k e^{k d}} .
$$

The substitution of these values in (13) gives:

$$
\begin{gathered}
Y-\frac{a I_{t}}{k} \operatorname{lgn} \frac{I}{I_{t}}-\frac{a}{k}\left(I_{t}-\frac{I}{e^{k d d}}\right)=(X-I)\left(\frac{a I_{t}}{k I}-\frac{a}{k e^{k d}}\right), \\
Y-\frac{a I_{t}}{k} \operatorname{lgn} \frac{I}{I_{t}}-\frac{a I_{t}}{k}+\frac{a I}{k e^{k \cdot d}}=\left(\frac{a I_{t}}{k I}-\frac{a}{k e^{k d d}}\right) X-\frac{a I_{t}}{k}+\frac{a I}{k e^{k d}},
\end{gathered}
$$

and after reduction of similar terms we obtain the general equation of the tangent:

$$
Y=\left(\begin{array}{l}
a I_{t} \\
k I
\end{array}-\frac{a}{k e^{k d}}\right) X+\frac{a I_{t}}{k} \operatorname{lgn} \frac{I}{I_{i}}
$$

At the point $A\left(I=I_{t}\right)$ it takes the form:

$$
Y=\left(\frac{a}{k}-\frac{a}{k e^{k d}}\right) X=\frac{a}{k}\left(1-\frac{1}{e^{k d}}\right) X
$$

and represents a straight line identical with the line given by equation (11).

In the same way the equation of the tangent at the point $B$ is found by making $I=I_{t} e^{k d}$ :

$$
Y=\left(\frac{a I_{t}}{k I_{t} e^{k d}}-\frac{a}{k e^{k d}}\right) X+\frac{a I_{t}}{k} \operatorname{lgn} \frac{I_{t} e^{k d}}{I_{t}}=a I_{t} d,
$$

which is the equation of a straight line identical with equation (12)

Let us deduce similar equations for the relation between the assimilation rate and the concentration $c$ of carbon dioxyde. We will consider again an algal suspension of the thickness $d$ illuminated 
from below by a light source of constant intensity $I_{0}$. A new complication arises from the necessity of taking into account two variable factors, viz. the carbon dioxyde concentration and the light intensity. Because of its gradual diminution from the bottom to the surface of the suspension it will be possible to find a certain level $z=h$ dividing this suspension into two layers. In the lower layer light is the factor in excess and the $\mathrm{CO}_{2}$ concentration is the limiting agent. On the other hand in the upper layer it is the intensity of iight which acts as the limiting factor. To obtain the rate of photosynthesis of the whole suspension we must calculate separately the rates for the two layers.

It is evident that in the lower layer the assimilation rate $F_{1}$ is proportional to the concentration of the carbon dioxyde and the thickness $h$ of the layer:

$$
F_{1}=b c h \text {, }
$$

$b$ being a proportionality factor. However $h$ is a function of $c$, for the greater is $c$ the greater must be the light intensity if light is not to become the limiting factor. If the carbon dioxyde concentration is increased from $c_{1}$ to $c$ the transition level $H$ (where the light intensity is $I_{H}$ ) will be lowered to a certain level $h$ with greater light intensity $I_{h}$. We shall assume that between $c, c_{1}, I_{h}, I_{H}$ there exist the relation: ${ }^{1}$

but from (1)

$$
\frac{c}{c_{1}}=\frac{I_{h}}{I_{H}} \quad \text { or } \quad \frac{c I_{H}}{c_{1}}=I_{h},
$$

$$
\frac{c I_{H}}{c_{1}}=I_{h}=I_{0} e^{-k \cdot h} \text { and } h=\frac{1}{k} \operatorname{lgn} \frac{I_{0} c_{1}}{I_{H} c} .
$$

The ratio $I_{0} / I_{H}$ is not a light intensity but a pure number, and therefore the expression $I_{0} c_{1} / I_{H}$ corresponds to a certain constant carbon dioxyde concentration and may be denoted by $c_{0}$ :

$$
c_{0}=\frac{I_{0} c_{1}}{I_{H}} .
$$

Then

$$
h=\frac{1}{k} \operatorname{lgn} \frac{c_{0}}{c},
$$

1 It may be pointed out that the relation (18) forms a new hypothesis which is not included in the fundamental assumptions (1) (4) and (5). 
substituting this value in (17) we obtain

$$
F_{1}=\frac{b c}{k} \operatorname{lgn} \frac{c_{0}}{c} .
$$

When deducing $F_{2}$ we may observe that the assimilation conditions are exactly the same as in the upper layer considered in the previous section of this paper, hence

$$
F_{2}=\int_{h}^{d} b I_{0} e^{-k z} d z=\left.\frac{-b I_{0}}{k} e^{-k z}\right|_{h} ^{d}=\frac{b I_{0}}{k}\left(\frac{1}{e^{k h}}-\frac{1}{e^{k d}}\right) .
$$

Substituting for $h$ the value (19) and considering that $e \operatorname{lognat} X=X$ we obtain

$$
F_{2}=\frac{b I_{0}}{k}\left(\frac{c}{c_{0}}-\frac{1}{e^{k d}}\right) \text {. }
$$

By a convenient change of the unit used to measure the light intensity we can write $I_{0}=c_{0}$, then:

$$
F_{2}=\frac{b}{k}\left(c-\frac{c_{0}}{e^{k d}}\right)
$$

Finally the assimilation rate of the whole suspension in relation to the carbon dioxyde concentration will be

$$
\begin{gathered}
F=F_{1}+F_{2}=\frac{b c}{k} \operatorname{lgn} \frac{c_{0}}{c}+\frac{b}{k}\left(c-\frac{c_{0}}{c^{k d}}\right) \\
\left(c_{0} e^{-k d} \leqslant c \leqslant c_{0}\right) .
\end{gathered}
$$

The functions (20) and (22) have a physiological meaning only for a certain range of carbon dioxyde concentration. Because of the physiological impossibility for $F_{1}$ to assume negative values it follows from (20) that $c_{0} \geqslant c$. For the same reason it results from (22) that

$$
c-\frac{c_{0}}{e^{k d}} \geqslant 0 \quad \text { or } \quad c_{0} e^{-k d} \leqslant c
$$

The meaning of $c_{n}$ can be read from (18a) and (1):

$$
c_{0}=\frac{I_{0} c_{1}}{I_{H}}=\frac{I_{0} c_{1}}{I_{0} e^{-k H}}=c_{1} e^{k H},
$$


if $H=o$ then $c_{0}=c_{1}$. When $H=o$ the transition level is situated at the bottom of the suspension, $c_{0}$ is therefore the minimum concentration for which, under given light conditions, the carbon dioxyde concentration ceases to be the limiting factor at all levels of the suspension.

The shape of the curve corresponding to function (23) is similar to that of function (9) (fig. 3). The coordinates of the initial and terminal points $A, B$ between which the curve has a physiological significance are:

$$
\begin{aligned}
& \text { point } A: \quad c_{A}=c_{0} e^{-k d}, \quad I_{A}=b c_{0} d e^{-k d} \\
& \text { point } B: \quad c_{B}=c_{0}, \quad I_{B}=\frac{b c_{0}}{k}\left(1-e^{-k d}\right) .
\end{aligned}
$$

It remains now to calculate the rate of photosynthesis for carbon dioxyde concentrations inferior or superior to the limits specified in (23). The condition $c<c_{0} e^{-k d}$ signifies that the $\mathrm{CO}_{2}$ concentration is the limiting factor in the whole suspension, or that $h=d$ in (17) and $F_{2}=o$. Hence

$$
F=F_{1}^{\prime}=b d . c, \quad\left(c<c_{0} e^{-k d}\right)
$$

i. e. for low carbon dioxyde concentrations the assimilation rate is proportional to the $\mathrm{CO}_{2}$ concentration. The meaning of the condition $c>c_{0}$ is that in the whole suspension the limitation is attributable to the insufficient light intensity; then $h=o$ and $F_{1}=o$, hence from (20):

$$
F=F_{2}=\int_{o}^{d} b I_{0} e^{-k z} d z=\frac{b I_{0}}{k}\left(1-e^{-k d}\right), \quad\left(c>c_{0}\right)
$$

i. e. for high dioxyde concentrations the assimilation rate is a constant independent of the $\mathrm{CO}_{2}$ concentration.

As in the first part of this paper it is easy to show that the straight lines represented by equations (25) and (26) are tangents to the curve (23) at the points $A$ and $B$ respectively. Indeed, if in the equation (13) for the tangent we make the following substitutions

$$
y=f(c)\left(\text { equ. 23), } \quad x=c, \quad f^{\prime}(x)=F^{\prime}=\frac{b}{k} \operatorname{lgn} \frac{c_{0}}{c}\right.
$$

we obtain the general equation of the tangent: 


$$
\begin{gathered}
Y-\frac{b c}{k} \operatorname{lgn} \frac{c_{0}}{c}-\frac{b c}{k}+\frac{b c_{0}}{k e^{k d}}=\frac{b}{k} \operatorname{lgn} \frac{c_{0}}{c}(X-c) \\
Y=\frac{b X}{k} \operatorname{lgn} \frac{c_{0}}{c}+\frac{b c}{k}-\frac{b c_{0}}{k e^{k d}} .
\end{gathered}
$$

At the point $A\left(c=c_{0} e^{-k d}\right)$ of curve (23) the equation (27) represents a straight line passing through the origin and identical with line (25):

$$
Y=\frac{b X}{k} \operatorname{lgn} \frac{c_{0} e^{k d}}{c_{0}}+\frac{b c_{0}}{k e^{k d}}-\frac{b c_{0}}{k e^{k d}}=b d X .
$$

The equation of the tangent at the point $B\left(c=c_{0}\right)$ is:

$$
Y=\frac{b X}{k} \operatorname{lgn} \frac{c_{0}}{c_{0}}+\frac{b c_{0}}{k}-\frac{b c_{0}}{k e^{k d}}=\frac{b c_{0}}{k}\left(1-e^{-k d}\right)
$$

and corresponds to a straight line parallel to the $X$-axis and identical with the line given by equation (26).

Finally let us compare the functions (9) and (23) expressing the assimilation rate in relation to the light intensity or the carbon dioxyde concentration. In order to make the similitude more apparent we shall denote by $X$ the variables $I$ and $c$ :

$$
\begin{aligned}
& F=\frac{a I_{t}}{k} \operatorname{lgn} \frac{X}{I_{t}}+\frac{a}{k}\left(I_{t}-\frac{X}{e^{k d}}\right) \\
& F=\frac{b X}{k} \operatorname{lgn} \frac{c_{0}}{X}+\frac{b}{k}\left(X-\frac{c_{0}}{e^{k d d}}\right)
\end{aligned}
$$

These two functions although showing some similitude are not identical. If however in (23) $c$ and $X$ are replaced by $X$ and $I_{t}$ respectively the function (23) is transformed into function (9). This result can be best understood from the following consideration: it results from assumption (18) that a dioxyde concentration is equivalent to a certain light intensity; if this intensity is measured in appropriate units we can write $c_{0}=I_{0}$ and substitute $I_{i,}$ or $c_{0}$ in (23):

$$
F=\frac{b c}{k} \operatorname{lgn} \frac{I_{0}}{c}+\frac{b}{k}\left(c-\frac{I_{0}}{e^{k d}}\right) .
$$


Now suppose that in (23a) we change the rôle of $I_{0}$ and $c$ and consider $I_{0}$ as the variable $X$ and $c$ as a constant, then

$$
F=\frac{b c}{k} \operatorname{lgn} \frac{X}{c}+\frac{b}{k}\left(c-\frac{X}{e^{k d}}\right),
$$

and it can be seen that functions (9) nad (28) have the same form. It follows from this similarity that in an algal suspension the function

$$
F=\frac{b c}{k} \log \frac{I}{c}+\frac{b}{k}\left(c-\frac{I}{e^{k d}}\right)
$$

represents the dependance of the assimilation rate upon the light intensity $I$ and the carbon dioxyde concentration $c$. If the object of our research is the influence of light on the assimilation rate $F, c$ is to be maintained constant and should be expressed in light intensities $\left(I_{t}\right)$. On the contrary, if we wish to study the influence of the $\mathrm{CO}_{\text {. }}$ concentration on $F, I$ is to be considered as a constant and should be expressed in terms of $\mathrm{CO}_{2}$ concentration $\left(c_{v}\right)$.

\section{III}

The functions given by the equations (9), (11), (12): (23), (25) (26) form the solution of the problem formulated in the introductory part of this paper. They were deduced from the principle of limiting factors and the additional assumption (18). Strictly speaking they are only valid for an uniform algal suspension; however, we may presume that they will retain their validity even when applied to multicellular plants, especially water plants. Owing to inequal distances from the light source and mutual shading the cells of a higher plant are illuminated with intensities ranging from feeble to strong, and therefore present some similarity with an algal suspension. Since the leaves of many aquatic plants are formed by one or two cell layers we may assume a nearly uniform carbon dioxyde concentration in all the cells. Therefore, this part of the paper, devoted to the experimental corroboration of the previous theoretical deductions, will not be limited to algal suspensions, but will use the experimental evidence obtained with multicellular plants by different writers.

However, since the values of the parameters $a, b, k, d, I_{t}$ and $I_{0}$ appearing in the functions (9) and (23) are not directly calculable 
from the experimental data it is necessary to transform these functions in an appropriate manner. Let:

$$
\begin{gathered}
\frac{k}{a I_{t}}=p, \quad \frac{1}{I_{t} e^{k d}}=q, \quad \operatorname{lgn} I_{t}=M \log I_{t}=M r, \quad \operatorname{lgn} I=M \log I \\
(\operatorname{lgn}=\text { lognat, } M=\text { logarithmic modulus 2, 302585 ...) }
\end{gathered}
$$

Then function (9) takes the form:

Similarly, if

$$
\begin{gathered}
F=\frac{M}{p} \log I-\frac{M r}{p}+\frac{1}{p}-\frac{q I}{p} \quad \text { or } \\
p F+q I+M r-M \log I-1=o .
\end{gathered}
$$

$$
\frac{k}{b}=p, \quad \frac{c_{0}}{e^{k d}}=q, \quad \operatorname{lgn} c_{0}=M \log c_{0}=M r, \quad \operatorname{lgn} c=M \log c
$$

the function (23) takes the form

$$
p F+q-M r c+M c \log c-c=o .
$$

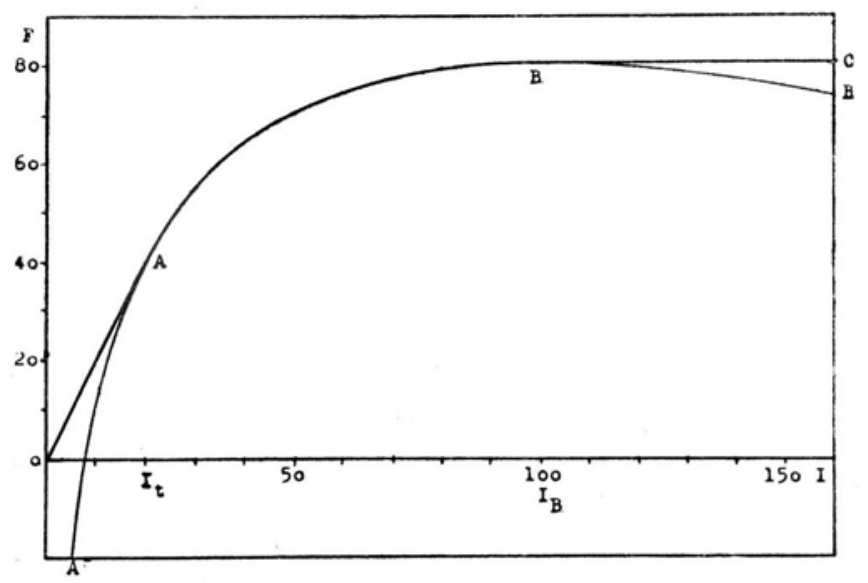

Fig. 2. Graphical representation of function (9). Abscissae: light intensities I, ordinates: assimilation rate $\mathrm{F}$. The curve $\mathrm{A}^{\prime} \mathrm{ABB}$ ' represents the function (9) (or (31)). It has, however, a physiological significance only along the arch AB. The tangents $\mathrm{OA}$ and $\mathrm{BC}$ to the curve at the points $\mathrm{A}$ and $\mathrm{B}$ correspond to the assimilation rates for light intensities below $I_{t}$ or above $l_{B}=I_{t} e^{k d}$. Thus the curve $\mathrm{OABC}$ represents the assimilation rate in relation to the light intensity. In the graph the coordinates of the points $A$ and $B$ are $I_{t}=I_{A}=20$, $F_{A}=40 ; I_{B}=100 ; F_{B}=80,47$. If the function (9) takes the form (31) the values of the parameters are: $\mathrm{p}=0,2, \mathrm{q}=0,01, \mathrm{r}=1,30103, \mathrm{Mr}=2,99573, \mathrm{~m}$ (angular coefficient of the tangent $\mathrm{OA})=2$. 
It can be shown that the values of $p, q$ and $r$ are calculable from the experimental data, which is done with more details in part IV. For the moment it will suffice to state that there are two slightly different ways for utilizing these data. In the first only the coordinates of the points lying on the arch $A B$ are used for calculating the parameters $p, q, r$. Then by comparing together the observed and the calculated values the agreement between the theoretical deductions and the experimental results can be checked. Moreover from the equations (11), (12), (25) and (26) it is possible to find the equations of the tangents $O A$ and $B C$ at the points $A$ and $B$ (fig. 2 and 3) and compare the values of their coefficients with the value calculated from the corresponding experimental data. A good agreement is a further corroboration of the theory.

In the second way of utilizing the experimental data first the equations of the lines $O A$ and $B C$ are found from these data, then the equation of the curve of the form (31) or (33) passing through given points and tangent to $O A$ and $B C$ is calculated and finally the

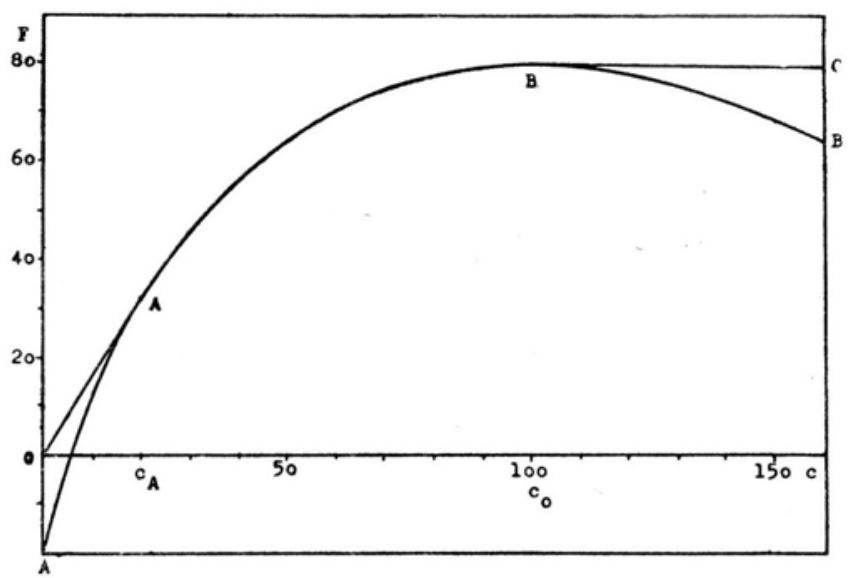

Fig. 3. Graphical representation of function (23). Abscissae: carbon dioxyde concentration c, ordinates: assimilation rate $\mathrm{F}$, Only the arch $\mathrm{AB}$ of the curve $\mathrm{A}^{\prime} \mathrm{ABB}$ ' has physiological significance. For carbon dioxyde concentrations below $c_{A}=c_{0} e^{-k d}$ or above $c_{B}=c_{o}$ the tangents $O A$ and $B C$ to the curve at the points $\mathrm{A}$ and $\mathrm{B}$ correspond to the assimilation rate. Thus the dependence of the assimilation rate upon the carbon dioxyde concentration is represented by the curve OABC. In the graph the coordinates of points $\mathrm{A}$ and $\mathrm{B}$ are: $\mathrm{c}_{\mathrm{A}}=20, \mathrm{~F}_{\mathrm{A}}=32,10 ; \mathrm{c}_{\mathrm{B}}=\mathrm{c}_{\mathrm{o}}=100, \mathrm{~F}_{\mathrm{B}}=80$. The value of the parameters of the modified form (33) of function (23) are: $p=1, q=c_{A}=$ $=20, \mathrm{r}=2, \mathrm{Mr}=4,60517, \mathrm{~m}=1,6005$. 
corresponding values of the calculated and observed ordinates are compared.

Only a part of the available evidence is presented here as a verification of our theoretical deductions. In choosing the evidence preference was given to experiments based on a greater number of simultaneous determinations of $F$ and $I$ or $F$ and $c$, and presenting little scatter. Since the number of researches on the action of the $\mathrm{CO}_{2}$ concentration on the assimilation rate is small there are only a few examples concerning the function (33) $F=f(c)$. It can be seen from the tables and the graphs ${ }^{2}$ that the agreement between the theory and the experimental results is good and is not limited to algal suspensions but also includes higher water plants and even leaves of land plants (cucumber). It is particularly apparent that a) the experimental data lying on the arch $A B$ show only slight deviations from the calculated values, (b) the observed $\left(m_{0}\right)$ and the calculated $\left(m_{c}\right)$ values of the angular coefficients of the tangent $O A$ do

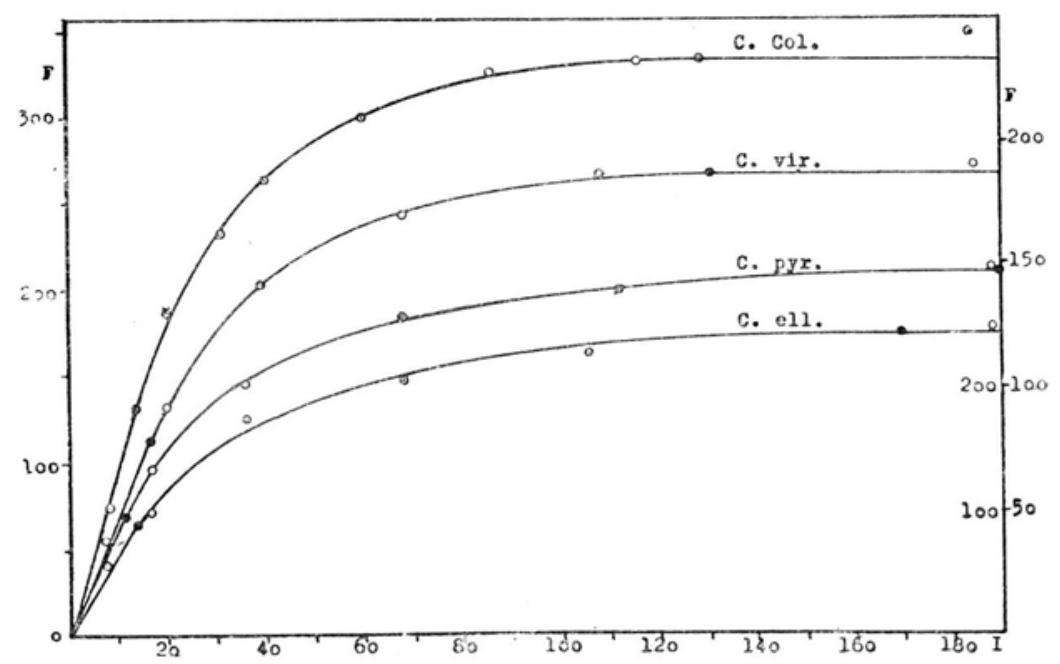

Fig. 4. Graphical representation of the data of tables 1-4: Chlorella vulgaris, var. viridis; Chl. vu!garis, Columbia strain; Chl. pyrenoidosa; Chl. ellipsoidea (W i n o k u r 1948). Abscissae: light intensities in meter candles $x$ 100; ordinates: assimilation rate in $\mathrm{cmm} \mathrm{O}_{2}$ per hour. At right scale for Chl. vulgaris, Columbia strain and Chl. pyrenoidosa; at left for Chl. viridis and Chl. ellipsoidea.

${ }^{2}$ In the graphs 4-7 the circles represent the experimental data, the black dots the transition points $\mathrm{A}$ and $\mathrm{B}$; the curves are the theoretical curves. of best fit corresponding to equations (31) or (33). 
not present greater discrepancies, c) the strict proportionality between the assimilation rate $F$ and $I$ or $c$ for feeble light intensities $I$ or small $\mathrm{CO}_{2}$ concentrations $c$, foreseen by our theory, is in agreement with the experimental evidence, d) in most cases the constancy of the assimilation rate for high light intensities or $\mathrm{CO}_{2}$ concentrations, also foreseen by our theory, is confirmed by experience.

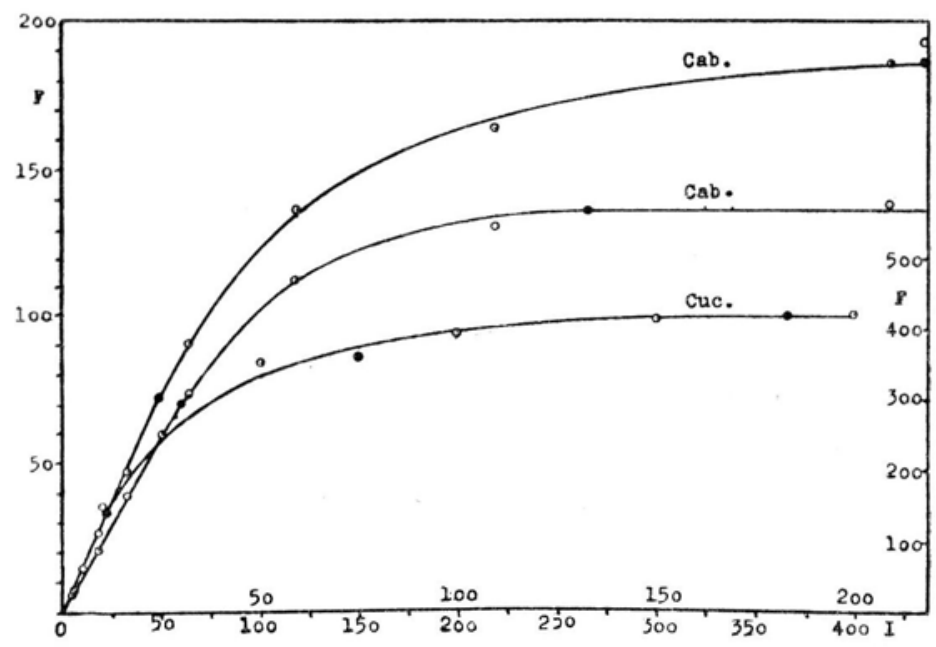

Fig. 5. Graphical representation of the data of tables 7, 8 and 12; Cabomba caroliniana ( $\mathrm{m}$ i t $\mathrm{h}$ 1937) and Cucumis sativus ( $\mathrm{G}$ a b $\mathrm{r} \mathrm{i} \in \mathrm{l} \mathrm{s}$ e $\mathrm{n}$ 1934). Abscissae: upper scale: light intensities in lux $\times 100$; lower scale in lux $\times 10$. Ordinates: $\mathrm{cmm} \mathrm{O}_{2}$ per hour (Cabomba), $\mathrm{mg} \mathrm{CO}_{2}$ per hour and $50 \mathrm{~cm}^{2}$ leaf surface (Cucumis). At left scale for Cabomba, at right for Cucumis.

In our opinion the good agreement found between our theore-tical deductions and the experimental results constitutes supplementary evidence supporting the validity of the fundamental assumptions, i. e. Blackmann's principle of limiting factors, on which our de.ductions are based. In the light of these deductions the smooth experimental curves appearing instead of two intersecting segments required by Blackmann's scheme, are not deviations from the principle of limiting factors (as assumed by numerous writers), but are a necessary consequence of the said principle.

Considering that the light employed in the different experiments was not monochromatic but consisted of a mixture of rays of different wave lengths (,white light"), the good agreement between the theory and the experimental results is somewhat unexpected. 
One would rather expect that the inequal absorption of the different radiations by the assimilatory pigments would lead to noticeable deviations from the theory. However, we may note that not the absorption as such but the photochemical efficiency of the different radiations is responsible for the rate of the carbon dioxyde assimilation. Researches on the dependence of quantum yield on the wave

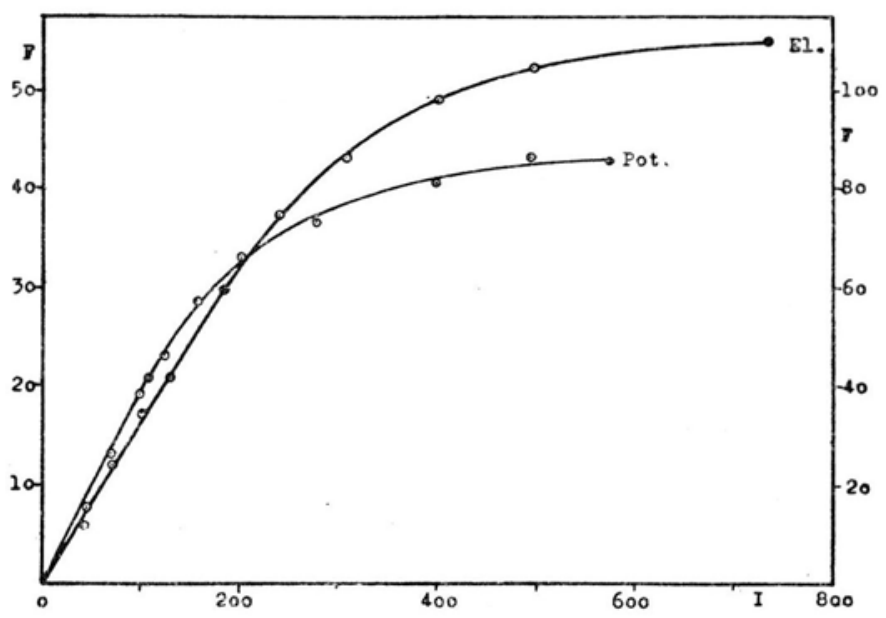

Fig. 6. Graphical representation of the data of tables 9 and 10: Elodea canadensis and Potamogeton sp. (G ó r s k i 1929). Abscissae: light intensities in arbitrary units, ordinates arbitrary units of oxygen ver min.

length (W a r b u r g 1923, $\mathrm{M}$ o o $\mathrm{r}$ e and $\mathrm{D} u \mathrm{~g} g \mathrm{~g} \mathrm{r}$ 1949) have shown that this yield varies but sligthly with the wave length and this explains the satisfactory agreement between theory and experiment found for white light. It is true that in the light that penetrates into the suspension not only its intensity but also its composition undergoes a change. In most cases however, this change car be neglected as it has only a small effect on the photosynthetic efficiency, the only thing that really matters being the gradual decrease of the light intensity in the suspension.

However, the greater discrepancies observed sometimes (for inst. table 2 and graph 4) for high light intensities (the experimental values being greater than the expected) are probably attributable to the inequal absorption and the photochemical efficiency of the different radiations forming the white light. The increase in $F$ observed when the light intensity is increased much above the value $I_{B}=I_{t} e_{k d}$ 
(the abscissa of point $B$ ) is presumably due to the fact that this increase supplies the higher layers of the suspension with radiations which, owing to strong absorption, do not normally reach these layers. The radiations in question are probably the strongly absorbed and photosynthetically very efficient red rays.

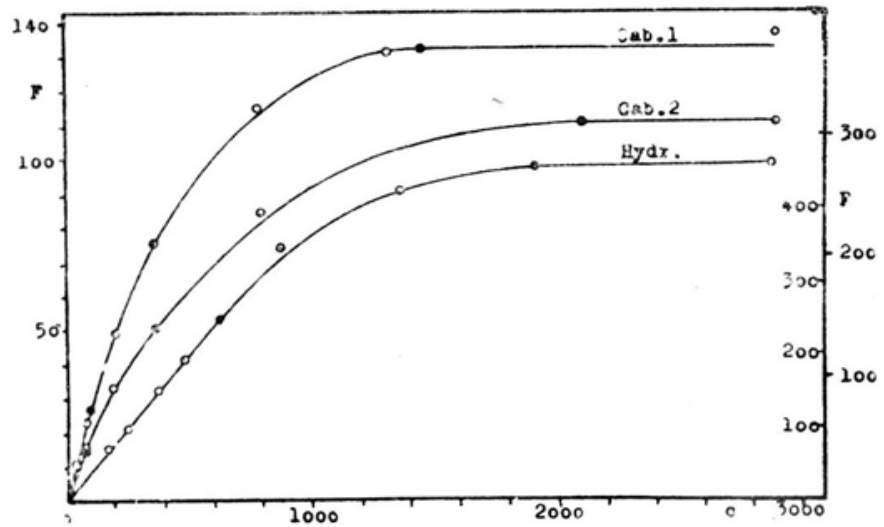

Fig. 7. Graphical representation of the data of tables 15, 16, 17: Cabomba caroliniana (S m i t h 1937) and Hydrilla verticillata (B o s e 1924). Abscissae: $\mathrm{CO}_{2}$ concentration in mols per liter $\times 10^{5}$ or mg per liter (Hydrilla) ordinates: $\mathrm{cmm} \mathrm{O}_{2}$ per hour. At left scale for Cabomba 1, at right for Cabomba 2 and Hydrilla.

Contrary to appearance there is no good agreement between theory and $\mathrm{W}$ a r b u r g's and $\mathrm{H}$ a $\mathrm{r}$ d e r's results recorded in tables 14 and 18. The calculation of the parameters leads to negative values of $q$ which means that the light intensity is the limiting factor even for minimal carbon dioxyde concentrations. This conclusion is however incompatible with the principle of limiting factors. As a probable cause of this discrepancy we may point out the fact that in both cases the $\mathrm{CO}_{2}$ concentrations are expressed in terms of bicarbonate concentration ${ }^{3}$, and it is known that the $\mathrm{CO}_{2}$ concentration of bicarbonate solutions does not increase strictly proportionally to the bicarbonate concentration. It is unfortunate that owing to the lack of more ample data it is impossible to verify the validity of function (23) for algal suspensions.

${ }^{3}$ In W a r b u r g's paper the $\mathrm{CO}_{2}$ concentrations were calculated from Dicarbonate concentrations. 
The previous deductions have shown that the curves representing the assimilation rate in relation to the light intensity or the carbon dioxyde concentration are formed by two segments connected by an arch. Let us now concentrate our attention on the factors upon which depends the length of the arch $A B$. The answer is given by formulas (10) and (24) indicating the values of the coordinates of the points $A$ and $B$. The distances between the end points of the abscissae of $A$ and $B$ are:

$$
\begin{aligned}
& D=I_{t} e^{k d}-I_{t}=I_{t}\left(e^{k d}-1\right) \text { for the function } \\
& D=c_{0}-c_{0} e^{-k d}=c_{0}\left(1-e^{k d}\right), \quad, \quad, \quad, \quad \text { (23) } F=f(c) .
\end{aligned}
$$

In these expressions $e^{k d}$ is always $>1, k$ (the absorption constant) and $d$ (the thickness of the algal layer) being two positive constants. It follows from these expressions that the length of the arch $A B$ will decrease with $k$ or $d$, i. e. with the density of the suspension or its thickness. For thin suspensions of small density the curves corresponding to $F=f(I)$ or $F=f(c)$ should consist of two segments connected by a very short arch. This is indeed the result obtained by $\mathrm{v}$ a $\mathrm{n}$ d e $\mathrm{n} \mathrm{H}$ o n e r $\mathrm{t}$ (1930) and $\mathrm{v}$ a $\mathrm{n}$ d e r P a u w (1932) in their study on the action of carbon dioxyde concentrations on photosynthesis. The assimilating layer, formed by the filamentous alga Hormidium was very thin, being only one cell thick. It must however be pointed out that in the same experiments, with light as the changing factor, the arch $A B$ was much longer.

\section{TABLES $1-18$}

In the columns 1 and 2 are recorded the experimental data, viz. the light intensity or the carbon dioxyde concentration and the assimilation rate, extracted from papers of different writers.

Column 3 gives the corresponding values of the assimilation rate calculated by means of functions (31) and (33).

$I$ - light intensity, $I_{t}=I_{A}$-transition value, (abscissa of point A), $I_{B}$ abscissa of point B (see fig. 2).

$c-$ carbon dioxyde concentration, $C_{A}$ and $C_{B}=C_{0}-$ abscissae of points

A and B (see fig. 3).

$F_{0}$ and $F_{c}$ - observed and calculated values of the assimilation rate $\mathrm{F}$.

$m_{0}$ and $m_{c}$ - observed and calculated values of the angular coefficient of the tangent at the point $\mathrm{A}$.

a. u. - arbitrary units,

mc. - meter candles,

$\mathrm{p}, \mathrm{q}, \mathrm{r}-$ see formulas (30) and (32) of the text.

(The data of table 17 are the sum of two similar series) 
1

Chlorella vulgaris var. viridis

(Winokur 1948)

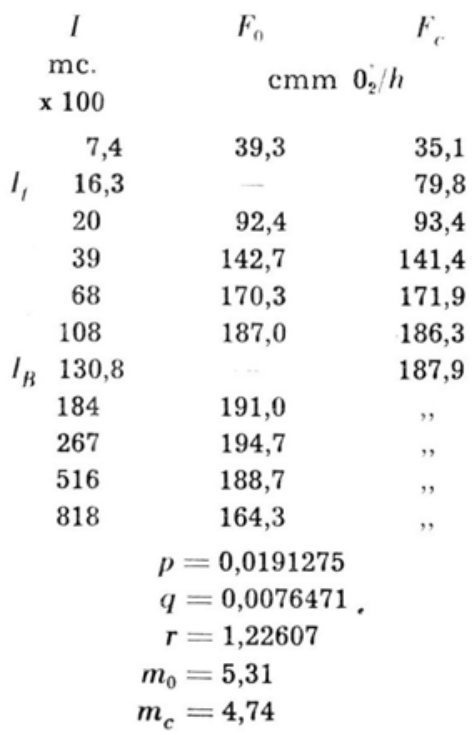

3

Chlorella pyrenoidosa

(Winokur 1948)

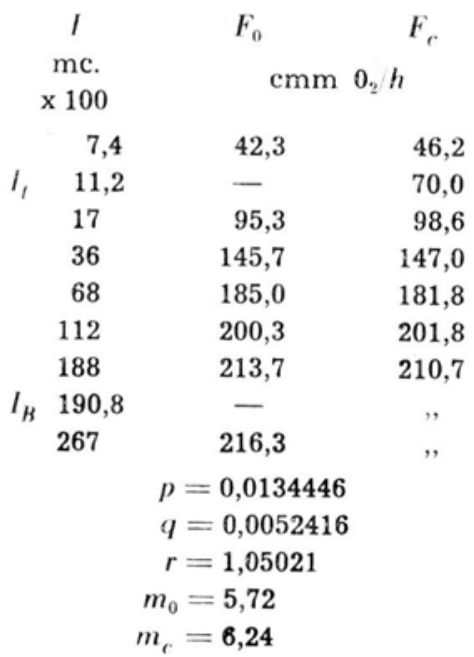

2

Chlorella vulgaris

(Columbia, Winokur 1948)

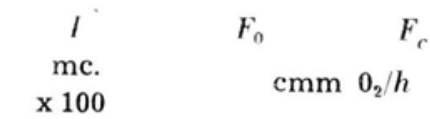

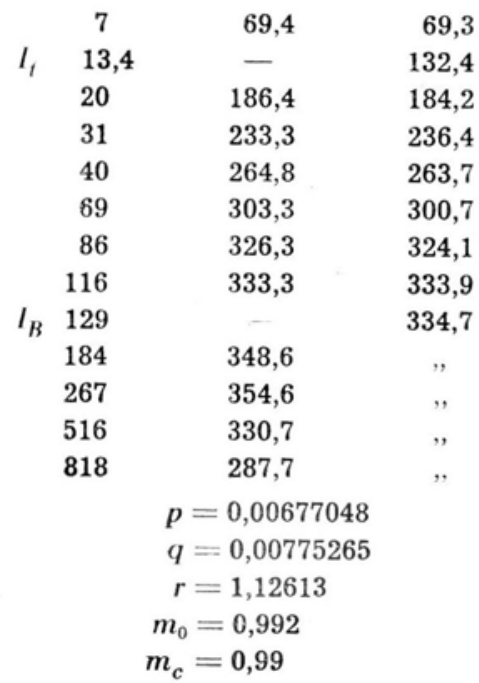

4

Chlorella ellipsoidea

(Winokur 1948)

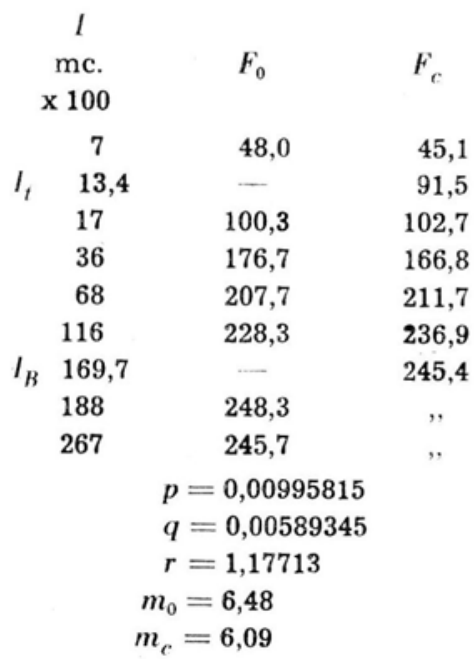


5

Chlorella sp. (Warburg 1919)

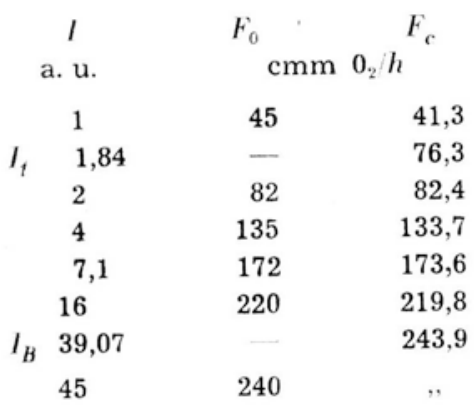

7

Cabomba caroliniana

(Smith 1937)

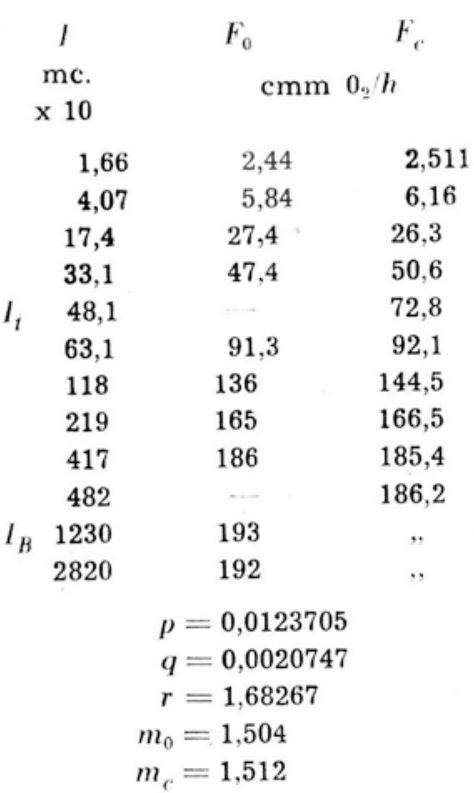

6

Fontinaiss sp. (Harder 1920)

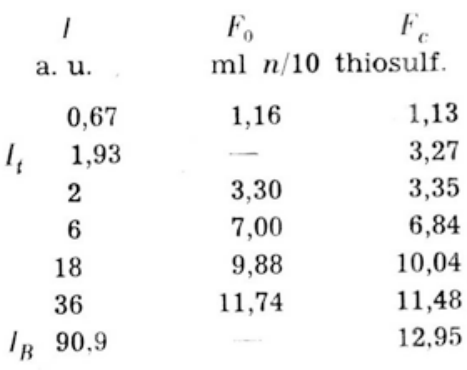

$$
\begin{aligned}
p & =0,3021 \\
q & =0,01104 \\
r & =0,28596 \\
m_{0} & =1,733 \\
m_{c} & =1,695
\end{aligned}
$$

8

Cabomba caroliniana

(Smith 1937)

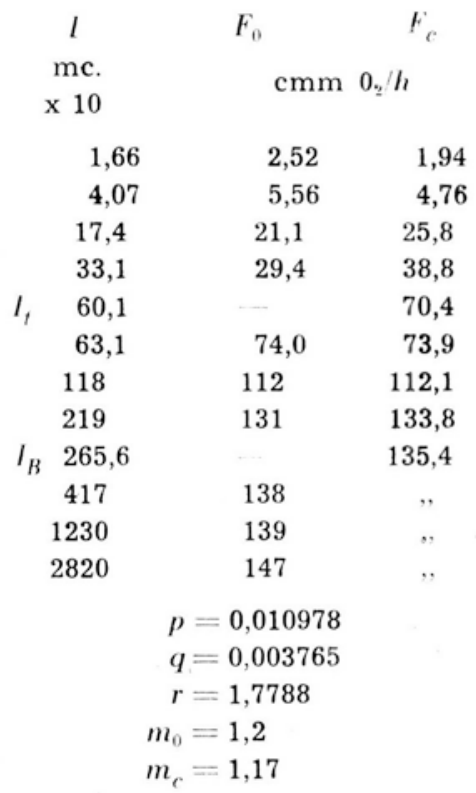


9

Elodea canadensis

(Górski 1929)



a. u.

47,7

72,5

103

134

181

If 183,2

240

306

403

497

$I_{B} \quad 736$

$$
\begin{aligned}
p & =0,0025241 \\
q & =0,0013599 \\
r & =2,26288 \\
m_{0} & =0,1654 \\
m_{c} & =0,1624
\end{aligned}
$$

\section{1}

Hydrilla verticillata

(Bose 1924)

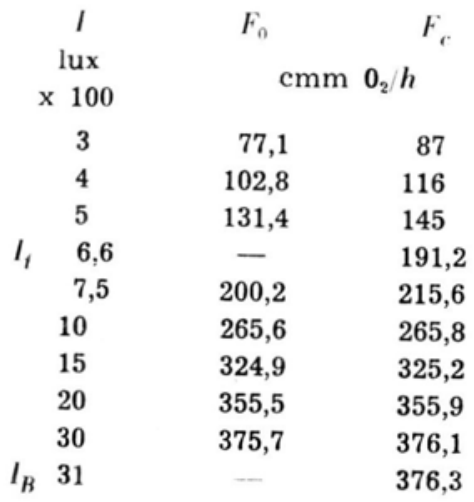

$$
\begin{aligned}
p & =0,0041268 \\
q & =0,0322162 \\
r & =0,81874 \\
m_{0} & =26,1 \\
m_{c} & =29,02
\end{aligned}
$$

10

Potamogeton lucens

(Górski 1929)

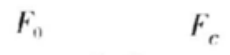

a. u.

a. u.

$\begin{array}{cccc} & 44,4 & 11,7 & 17,1 \\ & 69,4 & 26,1 & 26,8 \\ & 100 & 38,6 & 38,0 \\ I_{t} & 107,9 & & 41,7 \\ & 123 & 46,1 & 47,0 \\ & 156 & 57,2 & 56,3 \\ & 204 & 66,5 & 65,7 \\ & 278 & 73,6 & 75,0 \\ & 400 & 81,6 & 82,8 \\ & 494 & 86,4 & 85,2 \\ I_{B} & 575,4 & - & 85,8\end{array}$

12

Cucumis sativus (Gabrielsen 1934)

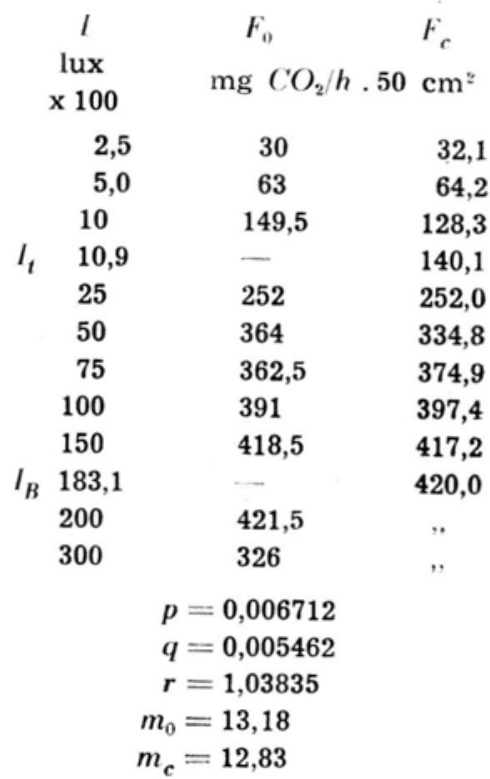


13

Hormidium sp.

(v. d. Honert 1930)

l

a. u.

$\begin{array}{clcc} & 1 & 28 & 28,1 \\ I_{t} & 1,81 & & \\ & 1,99 & 56 & 55,9 \\ & 3,08 & 76 & 77,1 \\ & 4,18 & 89 & 85 \\ & 6,18 & 100 & 99,7 \\ & 8,89 & & 103,5 \\ I_{R} & 13,2 & 104 & ,,\end{array}$

15

Cabomba caroliniana

(Smith 1937)

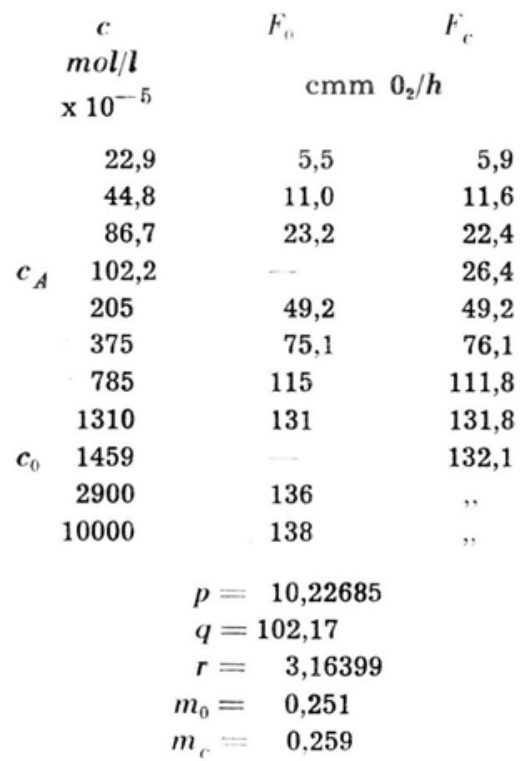

14

Fontinalis sp.

(Harder 1921)

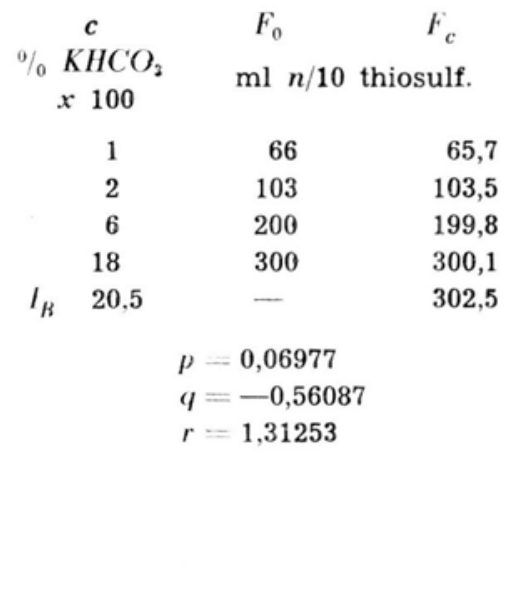

16

Hydrilla verticillata

(Bose 1926)

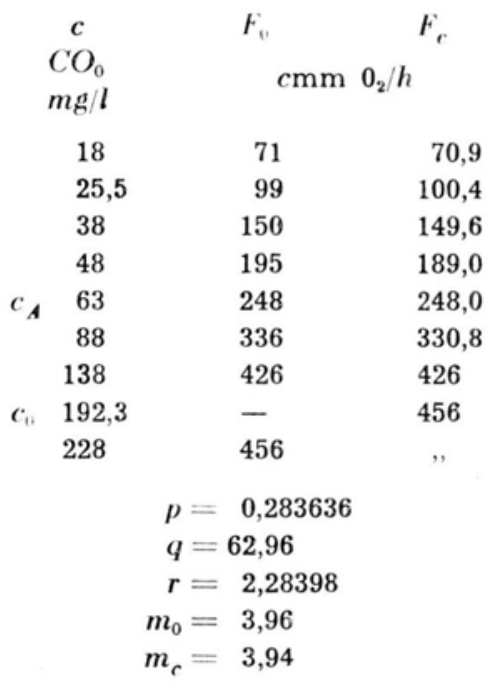


17

Cabomba caroliniana

(Smith 1939)

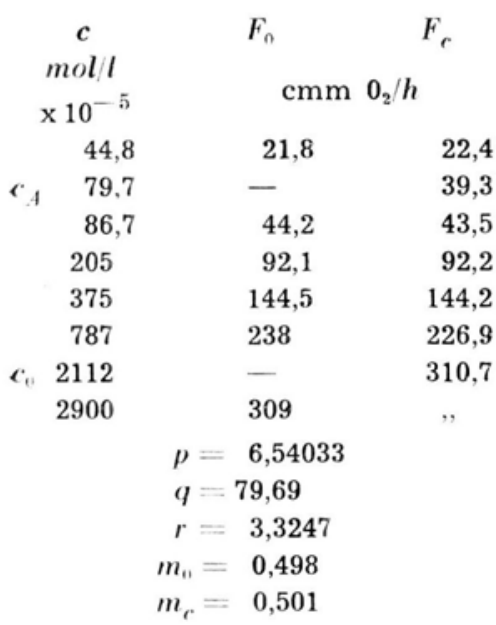

18

Chlorella sp.

(Warburg 1919)

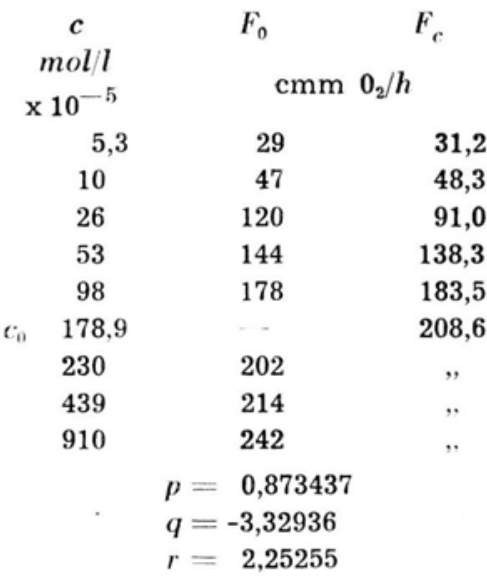

In this section the methods devised for the calculation of the parameters $p, q, r$ are presented in some detail.

1. The first is a least square method. The experimental data consist of $n$ pairs of values $I_{1}, F_{1}, I_{2}, F_{2}, \ldots . I_{n}, F_{n}$ from which all the values corresponding to the segments $O A$ and $B C$ are eliminated, since they are not lying on the arch $A B$ of the curve (31). The elimination is easily performed if the data are plotted on a graph. Values showing great deviations from the rest should be discarded. The least square treatment consists in finding values for $p, q, r$ which make the sum

$$
S(p F+q I+M r \quad M \log I-1)^{2}=S\left(D^{2}\right)
$$

a minimum. This condition is equivalent to

$$
\begin{aligned}
\frac{\partial S v^{2}}{\partial p} & =S|2(p F+q I+M r-M \log I-1) F|=0 \\
& =p S F^{2}+q S F I+M r S F-M S F \log I \quad S F=0 \\
\frac{\partial S v^{2}}{\partial q} & =S|2(p F+q I+M r-M \log I-1) I|=0 \\
& =p S F I+q S I^{2}+M r S I-M S I \log I-S I=0
\end{aligned}
$$




$$
\begin{aligned}
\frac{\partial S v^{2}}{\partial r} & =S|2(p F+q I+M r-M \log I-1) M|=0 \\
& =p S F+q S I+M n r-M S \log I-n=0 .
\end{aligned}
$$

From the above equations, which are linear with respect to $p, q, r$, these parameters are calculated by means of determinants. For this purpose we shall write the equations as follows:

$$
\begin{aligned}
& p S F^{2}+q S F I+M r S F=M S F \log I+S F \\
& p S F I+q S I^{2}+M r S I=M S I \log I+S I \\
& p S F+q S I+M n r=M S \log I+n
\end{aligned}
$$

We will show that

$$
p=\frac{M D_{p}}{D}, \quad q=\frac{M D_{q}}{D}, \quad r=\frac{D_{r}}{D}+\frac{1}{M},
$$

where

$$
\begin{gathered}
D=\left(n S F^{2} \cdot S I^{2}+2 S F . S I . S F I\right)-\left(S F^{2} \cdot S^{2} I+S^{2} F \cdot S I^{2}+n S^{2} F I\right) . \\
D_{p}=\left(S F I . S I-S F \cdot S I^{2}\right) S \log I+\left(n S I^{2}-S^{2} I\right) S F \log I+ \\
\quad+(S F . S I \quad n S F I) S I \log I, \\
D_{q}=\left(S F . S F I-S F^{2} \cdot S I\right) S \log I+(S F . S I-n S F I) S F \log I+ \\
\quad+\left(n S F^{2}-S^{2} F\right) S I \log I, \\
D_{r}=\left(S F^{2} \cdot S I^{2}-S^{2} F I\right) S \log I+\left(S F I . S I-S F \cdot S I^{2}\right) S F \log I+ \\
\quad+\left(S F \cdot S F I-S F^{2} . S I\right) S I \log I .
\end{gathered}
$$

Indeed, if we denote by $D$ the determinant

$$
D=\mid \begin{array}{lll}
S F^{2} & S F I & S F \\
S F I & S I^{2} & S I \\
S F & S I & n
\end{array}
$$

the value of $p$ is given by

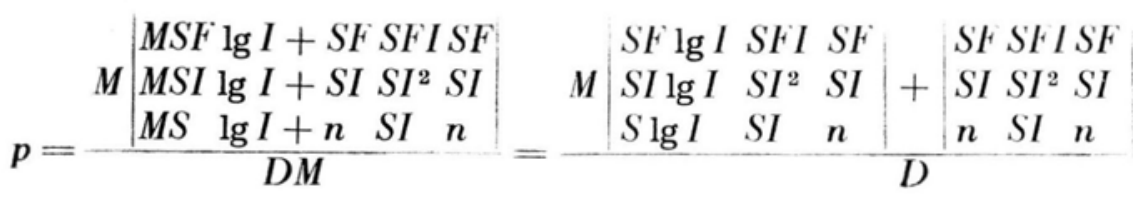


The second determinant in the numerator is nil since it has two identical columns. The value of the first, denoted by $D_{p}$, is given by the first formula (35), thus $p=M D_{p} / D$. In the same way it can be shown that $q=M D_{q} / D$, where

$$
D_{q}=\left|\begin{array}{l}
S F^{2} S F \lg I S F \\
S F I \quad S I \lg I S I \\
S F \quad S \quad \lg I n
\end{array}\right|
$$

The calculation of $D_{r}$ is slightly different:

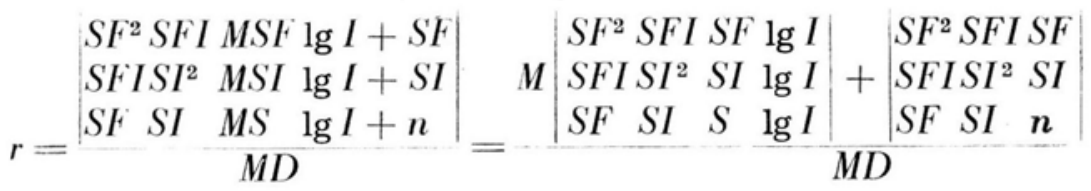

In the numerator the second determinant is equal to $D$, hence, if we denote the first by $D_{r}$, we obain:

$$
r=\frac{M D_{r}+D}{M D}=\frac{D_{r}}{D}+\frac{1}{M}=\frac{D_{r}}{D}+0,43429 \ldots
$$

Similar expressions are found for the parameters $p, q, r$ appearing in function (33). Since the calculations are the same we will limit ourselves to quote the principal steps. To the relations (34) correspond the equations:

$$
\begin{aligned}
& p S F^{2}+q S F-M r S F c=S F c-M S F c \log c \\
& p S F+q n-M r S c=S c-M S c \log c \\
& p S F c+q S c-M r S c^{2}=S c^{2}-M S c^{2} \log c
\end{aligned}
$$

and from them the parameters $\mathrm{p}, \mathrm{q}, \mathrm{r}$ are calculated:

$$
p=\frac{M D_{p}}{D}, \quad q=\frac{M D_{q}}{D}, \quad r=\frac{D_{r}}{D}-\frac{1}{M}
$$

where

$$
\begin{gathered}
D=\left(n S F^{2} . S c^{2}+2 S F . S c . S F c\right)-\left(S F^{2} \cdot S^{2} c+S^{2} F \cdot S c^{2}+n S^{2} F c\right), \\
D_{p}=\left(S F . S c^{2}-S c . S F c\right) S c \log c+\left(S^{2} c-n S c^{2}\right) S F c \log c+ \\
+(n S F c-S F . S c) S c^{2} \log c,
\end{gathered}
$$




$$
\begin{gathered}
D_{q}=\left(S^{2} F c-S F^{2} . S c^{2}\right) S c \log c+\left(S F . S c^{2}-S c . S F c\right) S F c \log c+ \\
\left.+S F^{2} . S c-S F . S F c\right) S c^{2} \log c, \\
D_{r}=\left(S F . S F c-S F^{2} . S c\right) S c \log c+(S F . S c-n S F c) S F c \log c+ \\
+\left(n S F^{2}-S^{2} F\right) S c^{2} \log c .
\end{gathered}
$$

2. If there are only 3 points $\left(I_{1}, F_{1}, I_{2}, F_{2}, I_{3}, F_{3}\right.$ or $c_{1}, F_{1}, c_{2}, F_{2}$, $c_{3}, F_{3}$ ) on the arch $A B$ the parameters are easily found by solving the following sets of linear equations:

or

$$
\begin{aligned}
& p F_{1}+q I_{1}+M r=M \log I_{1}+1 \\
& p F_{2}+q I_{2}+M r=M \log I_{2}+1 \\
& p F_{3}+q I_{3}+M r=M \log I_{3}+1
\end{aligned}
$$

$$
\begin{aligned}
& p F_{1}+q-M r c_{1}=c_{1}-M c_{1} \log c_{1} \\
& p F_{2}+q-M r c_{2}=c_{2}-M c_{2} \log c_{2} \\
& p F_{3}+q-M r c_{3}=c_{3}-M c_{3} \log c_{3}
\end{aligned}
$$

The correponding curves must exactly fit the given points. It is obvious that in this case the good agreement between the experimental results and the theory is a mathematical necessity and cannot be considered as an evidence corroborating our theory.

3. If $p, q, r$ are known it is possible to calculate:

a) the angular coefficient $m_{c}$ of the tangent $y=m x$ at the point $A$ and compare it with the observed value $m_{0}$,

b) the equation $y=F_{B}=$ const. of the tangent at the point $B$ and to compare it with the observed value.

The value $m_{c}$ of the angular coefficient of the tangent at the point $A$ of curve (31) is by (15) and (30):

$$
m_{c}=\frac{a}{k}-\frac{a}{k e^{k d}}=\frac{a I_{t}}{k I_{t}}-\frac{a I_{t}}{k I_{t} e^{k d}}=\frac{1}{p I_{t}}-\frac{q}{p}
$$

or finally

$$
\frac{1}{I_{t}}=e^{-\mathbf{M r}}=p m_{c}+q \quad \text { and } \quad m_{c}=\frac{e^{-M r}-q}{p}
$$


The observed value $m_{0}$ is found either from the formula $m_{0}=\frac{S(F I)}{S\left(I^{2}\right)}$ or from the mean of the ratios $F / I\left(m_{0}=\frac{S(F / I)}{n}\right)$, where $F$ and $I$ refer of course to pairs of experimental values lying on the segment $O A$. The expected value of $F_{B}$ is found by making in (31) $I=1 / q$, indeed (30), $1 / q$ is the abscissa $I_{B}=I_{t} e^{k d}$ of the point $B$.

or

$$
p F_{B}+1+M r=M \log 1 / q+1
$$

$$
p F_{B}-M \log q+M r=0 .
$$

The observed value of the constant in the equation $y=$ const. is simply the mean $\frac{F_{B 1}+F_{B 2}}{n}+\ldots . .+F_{B n}$ of all the experimental data of $F$ for which the abscissae are greater than the abscissa of the point $B$.

Similar calculations in the case $F=f(c)$ are given below. The equation of the tangent in $A$ is $y=b d c$ (25). Hence

$$
m_{c}=b d
$$

In this equality we must express $b$ and $d$ in terms of $p, q, r$. From $q=\frac{c_{0}}{e^{k d}}(32)$ it follows that $d=\frac{1}{k} \operatorname{lgn} \frac{c_{0}}{q}$, however, as $M r=\operatorname{lgn} c_{0}$ and $c_{0}=e^{\boldsymbol{v}_{r}}$, we have:

$$
d=\frac{1}{k} \operatorname{lgn} \frac{e^{M r}}{q}=\frac{1}{k}(M r-M \log q) .
$$

From (32) it follows also that $b=k / p$, substituting in (40) the above expressions we obtain:

$$
m_{c}=\frac{M r-M \log q}{p} \quad \text { or } \quad p m_{c}+M \log q-M r=0 .
$$

To express $F_{B}$ in terms of $p, q \cdot r$ we make $c=c_{0}$ in (23), $c_{0}$ being the abscissa of the point $B(24)$, but as $c_{0}=e^{\mathbf{M r}}$ we obtain:

and finally:

$$
p F_{B}+q-M r e^{M r}=e^{M r}-e^{M r} \operatorname{lgn} e^{M r}
$$

$$
p F_{B}+q-e^{M r}=0
$$


The observed values of $m_{0}$ and $F_{B}$ are calculated from the experimental data in the same way as in the case $F=f(I)$.

4. In the preceding methods the experimental data corresponding to the segments $O A$ and $B C$ were not used for the calculation of $p, q, r$. It is, however, possible to do so when starting from the assumption that the curve $F=f(I)$ or $F=f(c)$.

a) passes through one (or more) points lying on the arch $A B$.

b) is tangent to a given line $y=m x$ passing through the origin

c) is tangent to a given line $y=$ const. parallel to the $X$-axis. The following equations are equivalent to the above conditions:

$$
\begin{array}{ll}
\quad \text { for } F=f(I) & \multicolumn{1}{c}{\text { for } F=f(c)} \\
p F+q I+M r=M \log I+1 & p F+q-M r c=c-M c \log c \\
1 / I_{t}=e^{-M r}=p m+q & p m+M \log q-M r=0 \\
p F_{B}+M \log q+M r=0 & p F_{B}+q-e^{M r}=0
\end{array}
$$

where $I, c, F, F_{B}$ and $m$ are known and $p, q, r$ are the unknown parameters. Unfortunately there is no direct method for solving the above equations and therefore the general procedure used in such cases must be applied. It is however to cumbersome for practical purposes and will not be presented here.

The calculations become much simpler if the experimental data consist of $F_{B}$ and the coordinates $I_{1} F_{1}$ and $I_{2}, F_{2}$ of two points lying on the arch $A B$. The corresponding equations are

$$
\begin{aligned}
& p F_{B}+M \log q+M r=0 \\
& p F_{1}+q I_{1}+M r=M \log I_{1}+1 \\
& p F_{2}+q I_{2}+M r=M \log I_{2}+1 .
\end{aligned}
$$

We substract the second and the third equation from the first

$$
\begin{aligned}
& p\left(F_{B}-F_{1}\right)+M \log q-q I_{1}=-M \log I_{1}-1 \\
& p\left(F_{B}-F_{2}\right)+M \log q-q I_{2}=-M \log I_{2}-1 .
\end{aligned}
$$

If the following abbreviations are introduced

$$
f_{1}=F_{B}-F_{1} \text { and } \quad f_{2}=F_{B}-F_{2}
$$

the equations become:

$$
\begin{aligned}
& p f_{1}+M \log q-q I_{1}=-M \log I_{1}-1 \\
& p f_{2}+M \log q-q I_{2}=-M \log I_{2}-1 .
\end{aligned}
$$


The two equations are multiplied, the first one by $-f_{2}$, and the second one by $f_{1}$, and they are added together:

$$
\begin{aligned}
& -p f_{1} f_{2}-M f_{2} \log q+f_{2} q I_{1}=M f_{2} \log I_{1}+f_{2} \\
& p f_{1} f_{2}+M f_{1} \log q-f_{1} q I_{2}=-M f_{1} \log I_{2}-f_{1}
\end{aligned}
$$

$M\left(f_{1}-f_{2}\right) \log q+\left(f_{2} I_{1}-f_{1} I_{2}\right) q=f_{2}\left(M \log I_{1}+1\right)-f_{1}\left(M \log I_{2}+1\right)$

The final equation has either two or one (double) real solutions or no solutions at all. The solutions, if any, are readily found by successive approximations. A closer examination of the experimental data shows which of the two solutions is the right one. When $q$ is known $p$ and $r$ are immediately calculated from the second and third equation of the set (42).

A similar method applied to the set of equations

$$
\begin{aligned}
& p F_{B}+q-e^{M r}=0 \\
& p F_{1}+q-M r c_{1}=c_{1}-M c_{1} \log c_{1} \\
& p F_{2}+q-M r c_{2}=c_{2}-M c_{2} \log c_{2}
\end{aligned}
$$

leads to the equation

$\left(f_{1}-f_{2}\right) e^{M r}-\left(f_{1} c_{2}-f_{2} c_{1}\right) M r=f_{2} c_{1}\left(M \log c_{1}-1\right)-f_{1} c_{2}\left(M \log c_{2}-1\right)$,

where $f_{1}$ and $f_{2}$ have the same signification as in (43). Its solution is facilitated if $e_{M r}$ and $M r$ are replaced by $\mathrm{c}_{\mathrm{o}}$ and Mlog $\mathrm{c}_{0}$ (32).

\section{SUMMARY}

This paper is an attempt to elucidate the causes of the well known discrepancy appearing in photosynthesis between Blackmann's theory of limiting factors and the experimental results. It follows from this theory that the curves $F=f(I)$ and $F=f(c)$ representing the rate $F$ of carbon dioxyde assimilation in relation to the light intensity $I$ or the carbon dioxyde concentration $c$ should have the form of two intersecting segments. In reality the experimental curves $F=f(I)$ and $F=f(c)$ consist of two short segments connected by an arch of varying length and notably deviate from Blackmann's scheme. It is the aim of the present paper to demonstrate that the 
actual shapes of the curves $F=f(I)$ and $F=f(c)$ are a direct consequence of the principle of limiting factors, provided that the distribution of the light intensity in the assimilating plants or cells is taken into account.

In the first section the dependence of the assimilation rate upon the light intensity is examined with the help of a model formed by an uniform algal suspension illuminated from below. It is assumed that the intensity or the light pervading through the suspension diminishes according to Beer's law. It can be shown that there exist a certain level (transition level) dividing the suspension into two parts: in the lower part light is the factor in excess while in the upper part it plays the rôle of the limiting agent. Each part of the suspension is decomposed in very thin layers and the assimilation rate of each layer is calculated in conformity with the principle of limiting factors (formulas (4) and (5)). Then the contributions of all the layers are added and a function (formula (9)) relating the assimilation rate to the light intensity is obstained. In the same manner, (section II) the action of varying carbon dioxyde concentrations on the assimilation rate is examined and a function expressing the assimilation rate in relation to the carbon dioxyde concentration is found (formula (23)).

In the third section the theoretical deductions are checked with the experimental results drawn from various papers (by $\mathrm{B}$ o s e. Gabrielsen, Harder, van den Honert, Smith, $\mathrm{W}$ a r burg and $\mathrm{W}$ i n o k u r) referring to the dependance of the assimilation rate of algal suspensions and water plants upon the light intensity or the carbon dioxyde concentration. In most cases there is a satisfactory agreement between the theory and the experimental results. From this agreement the inference is drawn that the carbon dioxyde assimilation by green plants exactly conforms to the principle of limiting factors and that the actual shapes of the functions $F=f(I)$ and $F=f(c)$ far from presenting deviations form Blackmann's theory are its consequence.

Finally in the last section the methods elaborated for the calculation of the parameters appearing in functions (31) and (33), $F=f(I)$ and $F=f(c)$, are presented in detail. 


\section{REFERENCES}

B 1 a c k m a n n F. F. 1905. Optima and limiting factors. Ann. of Bot. 19: $281-95$.

B o s e J. C. 1924. The Physiology of Photosynthesis. London. 1-287.

B r i 11 i a n t B. A. 1949. Fotosyntez kak process żiznedejatelnosti rastenija Izdatelstwo Akademii Nauk SSSR. 1-159.

G a b r i e $1 \mathrm{~s}$ e n E. K. 1934. Untersuchungen über den Kohlenstoffhaushalt einer Gewächshauspflanze im Winter bei Tageslicht und mit elektrischer Zusatzbeleuchtung. Planta, 22: 180-89.

G ó r s k i F. 1929. Recherches sur les méthodes de mesure de photosynthèse chez les plantes aquatiques submergées. Acta Soc. Botanicorum Poloniae. 6: $1-29$.

$\mathrm{H}$ a $\mathrm{r}$ d e r R. 1921. Kritische Versuche zu Blackmanns Theorie der begrenzenden Faktoren bei der Kohlensäureassimilation. Jahrb. f. wiss. Botanik. 60: $531-71$.

va $\mathrm{n}$ d e $\mathrm{n}$ H o n e r t T. H. 1930. Carbon dioxyde assimilation and limiting factors. Rec. Trav. Bot. Néerl. 28: 149--286.

M o o r e W. E. \& D u g g a r B. M. 1949. Quantum efficiency of photosynthesis in Chlorella. In „Photosynthesis in Plants“ (monograph), Ames. Iowa, pag. $239-50$.

$\mathrm{v}$ a $\mathrm{n}$ d e r P a a u w F. 1932. The indirect action of external factors on photosynthesis. Rec. Trav. Bot. Néerl. 29: 497-620.

$\mathrm{S} \mathrm{m}$ i t h E. L. 1937. The influence of light and carbon dioxyde on photosynthesis. Journ. Gen. Physiol. 20: 807-30.

$\mathrm{S} \mathrm{m}$ i t h E. L. 1939. Limiting factors in photosynthesis: light and carbon dioxyde. Journ. Gen Physiol. 22: 21-35.

$\mathrm{S}$ p o e h r H. A. 1926. Photosynthesis. New York. 1-393.

$\mathrm{S}$ t i l e s W. 1926. Photosynthesis. London 1-268.

T s c he s n o k o v V. \& B a z y r i n a K. 1930. Die begrenzenden Faktoren bei der Photosynthese. Planta, 11: 457-62.

$\mathrm{W}$ a $\mathrm{r}$ b u $\mathrm{r} \mathrm{g}$ O. 1919. Über die Geschwindigkeit der photochemischen Kohlensäurezersetzung in lebenden Zellen. Biochem. Zeitschr. 100: $230-70$.

w a r b u r g O. \& N e g e 1 e i n E. 1925. Über den Einfluss der Wellenlange auf den Energieumsatz bei der Kohlensäureassimilation. Zeitschr. f. physik. Chemie, 106: 191-218. (Reprinted in: Warburg O.: Über die katalytischen Wirkungen der lebendigen Substanz, Berlin 1928).

W i n o k u r M. 1948. Photosynthesis relationships of Chlorella species, Amer. Journ. Bot. 35: 207-14. 